Voix et Images

voixetimages

\title{
Écrire le réel. Entretien avec François Charron
}

\section{Pierre Ouellet et Jacques Pelletier}

Volume 16, numéro 3 (48), printemps 1991

François Charron

URI : https://id.erudit.org/iderudit/200914ar

DOI : https://doi.org/10.7202/200914ar

Aller au sommaire du numéro

\section{Éditeur(s)}

Université du Québec à Montréal

ISSN

0318-9201 (imprimé)

1705-933X (numérique)

Découvrir la revue

Citer ce document

Ouellet, P. \& Pelletier, J. (1991). Écrire le réel. Entretien avec François Charron.

Voix et Images, 16(3), 382-392. https://doi.org/10.7202/200914ar d'utilisation que vous pouvez consulter en ligne.

https://apropos.erudit.org/fr/usagers/politique-dutilisation/ 


\section{Écrire le réel*}

\section{Entretien avec François Charron}

\section{par Pierre Ouellet et Jacques Pelletier, Université du Québec à Montréal}

\section{La venue à l'écriture}

V. \& I. - Qu'est-ce qui conduit un jeune homme à la poésie à la fin des années 60 ?

F. C. - Il y a de nombreux facteurs qui conduisent quelqu'un à la poésie. Il y a d'abord l'enseignement. En secondaire IV ou V, on a commencé à nous donner des cours de littérature. Moi je n'étais pas du tout familier avec la littérature, je ne viens pas d'un milieu où les livres traînent sur les tables ou dans les bibliothèques. Je viens d'une famille très modeste où on ne lit pratiquement pas. J'ai alors fait la connaissance d'un professeur passionné de littérature qui m'a donné le goût de lire. Jai découvert notamment des poètes, et les premiers que j'ai lus m'ont marqué énormément. Je me rappelle avoir lu Paul Verlaine, les Contemplations de.Victor Hugo, Baudelaire et Rimbaud. J'étais séduit avant tout par Verlaine. Du côté des Québécois, Émile Nelligan m'a beaucoup frappé. Donc, cet intérêt pour la littérature passait par un cours, par un professeur. J'ai également eu la chance d'avoir un ami, Roger Des Roches qui, à l'époque, était déjà écrivain. Il m'a donné le goût de la nouvelle littérature parce que pendant que moi je lisais Verlaine, Baudelaire, Nelligan ou Saint-Denys Garneau, il lisait déjà des poètes québécois et français plus contemporains, notamment les surréalistes. Il y a donc eu cette espèce d'interaction entre moi et Roger Des Roches qui me donnait le goût de lire encore plus et de commencer à écrire.

V. \& I. - Tu parles beaucoup de la tradition poétique, mais on a l'impression que tu as commencé à écrire de la poésie en te révoltant contre la poésie, notamment à travers les 18 assauts, enfin, contre une certaine idée de la poésie.

F. C. - C'est vrai, mais cela, c'est venu à l'âge de 18-19 ans. Avant d'en arriver là, il y a eu chez moi un profond besoin d'exprimer des émotions qui m'habitaient et que je n'arrivais pas à manifester. Je

* Entrevue réalisée en décembre 1990. Le texte reprend l'essentiel du contenu de cette rencontre, en respectant la formulation de l'auteur. 
n'avais pas les moyens, le médium pour exprimer ces émotions-là. La poésie était donc d'abord une façon d'exprimer des émotions.

V. \& I. - Cela veut dire qu'il y a eu une poésie plus lyrique, plus romantique avant ces textes-là?

F. C. - Oui, avant cela, j'ai fait des textes comme tous les étudiants en font, des sonnets, des textes très traditionnels, rimés, textes plus ou moins influencés par mes lectures. J'aurais pu én rester là, et arrêter d'écrire. J'avais envoyé ces textes d'adolescent à une revie que j'avais découverte en 1968, les Herbes rouges, et ils ont été refusés par Marcel Hébert qui m'avait dit que ces textes-là étaient "dégueulasses". Cela m'avait beaucoup impressionné. Et, tout compte fait, cela a été une chance pour moi. Je me suis dit: il a peut-être raison, la littérature ce n'est peut-être pas d'abord une affaire d'émotion, mais de langage. Et j'ai cessé d'écrire pendant un an après avoir rencontré Marcel Hébert. Je devais avoir 16-17 ans. Durant cette année où j'ai cessé d'écrire, j'ai lu beaucoup, je me suis intéressé davantage à la littérature québécoise. J'ai repris l'écriture au cégep où l'on a fondé une revue, Éther, qui publiait des textes d'étudiants, mais aussi d'écrivains: Nicole Brossard, Raoul Duguay, Gilbert Langevin, Denis Vanier, etc.

V. \& I. - Quand as-tu commencé à prendre une distance ironique par rapport à la poésie avec un grand $\mathrm{P}$ ?

F. C. - À l'époque, on tentait de renouveler l'approche du phénomène littéraire. On avait le projet de développer une science du texte, une science de la littérature. J'ai commencé un peu à m'intéresser à ces tentatives à travers Tel Quel. Je lisais Foucault, Barthes, Derrida, des gens qui travaillaient sur la littérature mais à partir de différents points de vue: la philosophie, la psychanalyse, la linguistique, la sémiologie, etc. Je m'intéressais aussi beaucoup à la philosophie, qui a été pour moi une découverte extraordinaire. Une révélation.

V. \& I. - Ce que tu évoques-là n'est-il pas un peu lié à la période du mai 68 français, de l'agitation étudiante'?

F. C. - Au cégep où j'étais, il y avait beaucoup d'activités, le milieu étudiant était très actif sur le plan politique; il y avait des groupes d'étudiants qui étudiaient la philosophie, la politique. Et les cours de philo étaient très sérieux: on étudiait les textes d'Althusser, les essais philosophiques de Mao Tse-Toung, etc. En littérature aussi, il y avait quelques professeurs qui lisaient un peu ce qui se passait de plus actuel en France. Cela m'a beaucoup marqué, et influencé. C'est à cette époque que j'ai lu un numéro de Liberté produit par des jeunes étudiants de l'Université du Québec, des étudiants qui, justement, s'intéressaient à la littérature, à la sémiologie, et avec qui j'ai voulu entrer en contact parce que j'avais l'idée de fonder une 
revue littéraire. C'est comme ça que j’ai rencontré des gens comme Gaétan Saint-Pierre et Paul Rompré avec qui j’ai fondé la revue Stratégies.

\section{La déconstruction, Tel Quel}

V. \& I. - À ce moment-là ta pratique est vraiment liée à la déconstruction?

F. C. - Oui, dans mes premiers textes, ma pratique est très collée à la pensée de la déconstruction, très près de celle de certains auteurs de Tel Quel, dont Denis Roche, qui m'ont beaucoup influencé, surtout dans mon tout premier livre. En 1971, j'ai fait un voyage déterminant dans le cadre des échanges franco-québécois pour la jeunesse. J'ai rencontré beaucoup d'écrivains en France qui étaient impliqués dans des revues, Jean-Pierre Faye, Philippe Sollers, Marcellin Pleynet, Guillevic, etc. C'est durant ce voyage-là que j'ai découvert Bakhtine, notamment, et que je me suis rapproché un peu plus de la problématique du carnaval. Au retour, j'ai décidé de faire un travail de déconstruction de la poésie bien-pensante, cette poésie qui, de mon point de vue, véhiculait des idées qui faisaient partie de ce que j'appelais une conception idéaliste, métaphysique du monde. Pour moi, à l'époque, cette conception-là retardait le progrès non seulement de la pensée mais le progrès social en général. Et je voulais faire des textes qui se situent dans la ligne du progrès, qui avancent en parallèle avec les mouvements sociaux les plus d'avantgarde, les plus à gauche, les mouvements qui veulent transformer la société dans le sens d'un mieux-vivre, d'un mieux-être, d'une plus grande liberté.

V. \& I. - Mais ton mode d'intervention dans le social en tant qu'écrivain était radicalement différent de celui de tes aînés de Parti pris et de l'Hexagone. Étais-tu conscient de cela à l'époque?

F.C. - J'en étais un peu conscient. Je connaissais le travail de Gérald Godin. J'avais surtout lu et aimé le travail de Paul Chamberland, notamment L'afficheur hurle qui était, selon moi, un texte carnavalesque important. Évidemment, en même temps, je travaillais beaucoup sur le langage en tant que tel et ce que j'attaquais, ce n'était pas directement une classe sociale ou des idées immédiatement politiques. J'attaquais avant tout une littérature qui m'apparaissait comme indéfendable sur le plan social et sur le plan philosophique, une littérature qui servait les intérêts d'une minorité, qui empêchait le développement d'une plus grande liberté, l'émergence d'une société plus juste, plus ouverte.

V. \& I. - Ce travail était donc sous-tendu par une prise de position carrément politique? 
F. C. - Je voulais alors vraiment me mettre au service de ce que j'appelais la "cause" politique. Pour me mettre au service de cette cause-là, je me suis rendu compte qu'il fallait que mes textes deviennent plus lisibles, qu'ils abordent d'une façon beaucoup plus directe la question politique. J'ai donc utilisé dans mes textes un langage beaucoup plus près de la parole, un langage de dénonciation stigmatisant des situations sociales intolérables. Et j'ai emprunté beaucoup au discours marxiste pour que mes textes soient plus accessibles non seulement aux lecteurs de poésie mais aussi aux militants politiques. Je pensais que je pouvais rendre des services à la "cause" " et que les militants politiques pourraient me lire et apprécier mon travail. Ce qui n'a absolument pas été le cas.

\section{V. \& I. - Pourquoi?}

F. C. - On aurait voulu que j'écrive de la poésie un peu comme on parle, que je m'adresse directement à certaines personnes et que je dénonce les situations sociales intolérables. On n'acceptait pas la dimension pulsionnelle de mes textes, celle qui ne renvoyait pas à un sens immédiat très facile à comprendre. Or, j'essayais d'exprimer une conception du monde qui n'était pas uniquement la mienne, qui était partagée par d'autres tout en voulant rendre compte de mon monde intérieur: je ne voulais pas m'effacer totalement au profit du politique. J'étais incapable de me censurer complètement.

\section{La fin des certitudes}

V. \& I. - Quand au juste as-tu pris une distance explicite par rapport à la "cause"?

F. C. - Vers la fin des années 70 , il y a eu un début de démembrement des mouvements communistes et socialistes, il y a eu beaucoup de déception notamment par rapport à la Chine, et il y a eu la découverte des goulags dans l'œuvre de Soljenitsyne. Par ailleurs, sur lé plan personnel, je me rendais compte qu'on pouvait se servir de tout, et même du discours politique pour masquer les choses, pour se mentir et pour mentir aux autres. J'ai pris conscience qu'il était important pour moi de prendre une distance par rapport à ce langage politique-là qui était en train de devenir une nouvelle forme de censure.

V. \& I. - Est-ce que la "vie privée" a compté pour beaucoup dans cette évolution?

F. C. - La vie privée, c'est quelque chose qui déborde le cadre du politique. Ça concerne toute la vie affective, la vie intérieure, la douleur, la joie. J'étais alors préoccupé de savoir pourquoi et comment vivre plus intensément. Et la politique ne pouvait pas répondre à cela. La vie est toujours plus que l'image ou que l'idée 
qu'on s'en fait, qu'elle soit politique ou autre. Je suis convaincu que la vie est belle parce qu'en quelque part elle nous échappe.

V. \& I. - Est-ce que ton expérience, sur ce plan, est représentative de ce qui se passe depuis le début des années 1980, c'est-à-dire un retour à l'individualité?

F. C. - Je pense que oui. Évidemment, il y a toujours des courants qui se manifestent, mais je pense qu'effectivement les jeunes sont retournés à des problèmes qui sont liés beaucoup à la vie personnelle, à la vie affective. On est revenus à ce que jappelle la parole, par opposition au texte, à une parole plus simple qui fait beaucoup moins directement référence aux sciences humaines. On s'est peut-être rapprochés d'une certaine spiritualité aussi.

V. \& I. - Ce dont témoignait, entre autres, le recueil Qui a peur de l'écrivain? en 1984. Comment vois-tu le débat qui a alors eu lieu, avec le recul du temps?

F. C. - Je crois qu'il y a eu alors une réaction à ce que j'appelle le nouveau. On a fait peur à beaucoup de gens. En soulevant la question du religieux, en soulevant aussi celle de la douleur. Et certains ne voulaient pas en entendre parler; pour eux, la douleur c'était réactionnaire, anti-scientifique, ça ne servait à rien. Ça ne servait surtout pas à défendre une cause. Une certaine avant-garde littéraire, à l'époque, a donc été très scandalisée qu'on puisse aborder le terrain de la douleur humaine en tant que telle.

V. \& I. - N’y avait-il pas là aussi un conflit entre deux tendances littéraires?

F. C. - Oui, il y avait une lutte entre deux factions, mais moi, je ne voulais pas défendre une faction, je défendais mon droit à la parole tout simplement. Un certain nombre d'écrivains prétendaient dicter ce qui doit se faire en poésie. Quand on est arrivés avec ces nouveaux textes-là, ils se sont peut-être sentis un peu menacés dans leur hégémonie, dans leur pouvoir. Donc, il y a eu indirectement une lutte de pouvoir. Moi, le seul pouvoir que je voulais, c'était celui de m'exprimer le plus librement possible. Je ne voulais pas m'empêcher d'écrire ce qui me venait et je ne voulais surtout pas nier mes intuitions par rapport à la poésie, au réel, à ma propre vie. Je pense que ces gens-là trouvaient que tout ce qui est du domaine de l'intuition était négligeable ou même dangereux. On a fait courir toutes sortes de rumeurs à mon sujet... C'était rendu que j'allais à l'église, que je priais, que j'allais quasiment communier. Certains, je pense, se sont sentis menacés dans leur pouvoir de représentation.

V. \& I. - Il y avait là aussi une défense de la poésie pour elle-même?

F. C. - Je défendais l'idée de poésie, parce que l'idée même de poésie était vue comme quelque chose de dépassé. C'était quelque chose de 
tout à fait indéfendable. On en avait beaucoup contre le lyrisme aussi, à l'époque, et moi j'ai toujours été assez lyrique...

V. \& I. - La mort de la NBJ récemment, qu'est-ce que ça signifie? La fin de la modernité?

F. C. - Je ne sais pas, mais c'est sûrement l'échec d'une tentative de refoulement. Ça, j'en suis très heureux. Je pense qu'à la NBJ, on a fait des choses essentielles et importantes, mais que dans les dernières années, on ne publiait que des textes qui se renvoyaient les uns aux autres.

V. \& I. - Sa fin marque l'essoufflement d'un courant qui a quand même été important pendant 15 ans?

F. C. - Je crois que oui, la fin d'un courant qui ne voulait se renouveler d'aucune façon.

V. \& I. - Est-ce 'qu'on peut interpréter ce que tu dis comme une critique de la modernité? Est-ce que tu accepterais de dire que ton travail s'identifie, par conséquent, à une sorte de post-modernité? Ça t'énerve, ce mot-là?

F. C. - Non ça ne m'énerve pas, mais les catégories. sont toujours un peu embarrassantes. La modernité, c'était devenu comme une chapelle; elle relevait beaucoup plus d'un esprit de famille que d'un esprit de recherche, d'expérience et d'exploration. Moi, je voulais continuer à explorer et je pense que je suis toujours resté quelqu'un qui explore, qui recherche, qui déplace des choses et je trouvais qu'on tournait en rond. Je trouvais qu'il n'y avait rien qui bougeait, ça m'ennuyait au plus haut point.

V. \& I. - En fait, on a l'impression qu'à cette époque tu assumes sans culpabilité, sans fausse pudeur le lyrisme qui parcourt tes textes depuis le début.

F. C. - Oui absolument. J'ai dépassé la culpabilité que je ressentais jusqu'alors à l'endroit du "père" politique. J'ai décidé de ne pas me censurer ou me taire parce que ce n'est pas bien d'un point de vue politique.

\section{L'écriture: une vocation, une pratique}

V. \& I. - À quand remonte ton engagement total dans l'écriture?

F. C. - En 1977, après avoir quitté l'enseignement. Je me suis rendu compte que je ne faisais qu'écrire et que ça allait relativement bien, que ma vie, c'était ça: écrire. Je n'ai pas décidé de devenir écrivain. Je ne me suis jamais vraiment posé de questions par rapport à ce qui pouvait ou devait m'arriver. J'essaie de laisser aller le plus les choses, de ne pas trop diriger tout ça. Je dis souvent en blaguant que c'est peut-être la seule chose que je sais faire; je ne la 
fais d'ailleurs pas toujours très bien. Mais en somme, je me débrouille pas trop mal...

V. \& I. - As-tu comme projet de pratiquer d'autres genres littéraires que la poésie?

F. C. - Oui, j'ai dans la tête un projet de journal, de cahier de notes où la poésie rejoindrait le travail de réflexion, le travail de la pensée. Ca m'intéresserait de mettre au clair tout ce que j'ai vécu depuis une dizaine d'années. Mais j'ai une préférence pour la poésie, parce qu'il y a quelque chose de spontané dans cette forme de création qui correspond à ma sensibilité personnelle. J'ai appris à être patient mais il reste que jaime bien que les choses se fassent le plus rapidement possible. Et la poésie me permet de faire plus rapidement que le roman les choses que je tiens à faire.

V. \& I. - La poésie est donc liée pour toi à une forme d'immédiateté. Cela signifie-t-il que tes textes sont intouchables, que tu ne les retravailles pas, une fois qu'ils sont produits dans la spontanéité du geste?

F. C. - Non, pas du tout. Plus mes textes sont simples, plus ils sont travaillés. Je travaille beaucoup plus mes textes aujourd'hui que je les travaillais il y a une dizaine d'années par exemple. Parfois, bien 'sûr, il y a des vers ou des phrases qui nous sont donnés. Ça, c'est extraordinaire. C'est mon plus grand plaisir: écrire un court poème ou une phrase et me dire: oui, c'est exactement ça que je veux. Mais habituellement, il y a un travail qui se fait; et je peux écrire cinq, six, dix versions du même texte.

V. \& I. - Comment, concrètement, travailles-tu un poème? Quelles sont les situations qui déclenchent l'écriture?

F. C. - D'abord, il y a mon travail d'écriture en tant que tel. Les livres que j'écris s'influencent les uns les autres, me font avancer. Habituellement, il y a aussi ce que j'appellerais des idées générales qui me trottent dans la tête. Dans mes derniers livres, par exemple, j'ai voulu mettre en situation des événements très banals, très quotidiens, et j'ai essayé de mettre ça en relation avec une dimension beaucoup plus intérieure, plus philosophique. Au point de départ donc des idées très générales. Mais le texte est fait vraiment au moment de l'écriture. Il est produit par la main qui trace.

V. \& I. - Le texte vient-il d'un seul tenant ou en pièces détachées? Comment, à partir de notations sur la vie quotidienne, d'observations visúelles, le texte intègre-t-il des éléments plus abstraits?

F. C. - Habituellement ça vient ensemble mais pas toujours. Quand jutilise une série de phrases qui renvoient au quotidien, qui sont de l'ordre du constat, je fais intervenir à un moment donné une phrase plus philosophique, plus abstraite, pour ouvrir l'espace, de telle sorte 
que les constats renvoient à autre chose, créent une atmosphère, un état d'esprit différent. Mais il arrive que ça vienne ensemble.

V. \& I. - Si ça vient ensemble, il y aurait donc une partie du travail qui ne serait pas concertée, qui serait de l'ordre de l'inconscient?

F. C. - C'est toujours concerté. Il y a toujours des liens entre les, éléments, même s'ils sont parfois éloignés ou obscurs. Ce qui fait qu'un poème fonctionne ou pas, c'est qu'il crée une atmosphère. Et pour cela, il faut qu'à travers l'arrangement des phrases un climat apparaisse. Sinon, ce n'est pas intéressant, ca devient un travail purement énumératif, ce n'est plus de la littérature.

V. \& I. - Et la création interviendrait dans la rencontre, la contamination des deux discours, celui de la vie quotidienne et celui, disons, de la philosophie? Le quotidien devient ainsi matière à penser, et la pensée devient une affaire de tous les jours.

F. C. - Effectivement, ça se passe un peu comme ça.

V. \& I. - Ça fait penser à la métaphore surréaliste: il s'agit de rapprocher deux choses très éloignées qui fassent sens, mais cela résulte du travail sur les mots alors que tu le fais, toi, sur des énoncés complets: tu rapproches des plans de pensée très éloignés les uns des autres et ça produit l'effet poétique. En cela, c'est différent de la poésie de Philippe Haeck, par exemple, qui parle aussi de la vie quotidienne mais en s'y tenant au plus près, sans en dégager le sens métaphysique. C'est la dimension spirituelle qui distinguerait ton 'travail du sien?

F. C. - C'est beaucoup moins narratif chez moi, je pense. Et c'est vrai que la dimension spirituelle est moins forte chez Haeck.

V. \& I. - À quand justement remonte chez toi cette préoccupation du spirituel? Quand intervient-elle dans ton travail d'écriture?

F. C. - C'est difficile à situer précisément. Un texte l'annonce en tout cas, c'est sûr, c'est Feu. Il y a là un éclatement épouvantable. C'est un texte très important pour moi qui annonce Blessures, un texte formaliste, difficile d'accès, qui parle vraiment des blessures du sujet, de ses difficultés à vivre, à parler, à assumer ses échecs, à continuer à écrire aussi. Â ce moment-là, je n'étais pas sûr de pouvoir continuér à écrire. Et d'une certaine manière ce texte m'a sauvé du naufrage. Mais le texte qui ouvre vraiment la problématique dans laquelle je suis encore aujourd'hui, c'est Mystère. Il y a dans ce texte un effort de simplification, de lisibilité, et j'y mets en place le thème religieux et métaphysique: je parle de Dieu, du monde, du mystère, etc. Ce texte pour moi annonce tout ce qui va venir par la suite.

V. \& I. - Quand es-tu entré en contact avec Borduas? 
F. C. - Quand j'ai fait de la peinture, le Refus global a été un texte important pour moi comme peintre. Par ailleurs, c'est un écrit qui remet en question la pensée nationaliste fermée, conservatrice, qui empêche d'aller vers l'autre, de rêver, de créer... Ça demeure pour moi un texte très libérateur, qui questionne l'idée d'idéologie, qui' essaie de montrer que les idéologies - qu'elles soient de droite ou de gauche - essaient toujours d'étouffer ce que le texte appelle l'élan créateur, les mystères objectifs... C'est un texte qui lutte contre toutes les formes de peur et en cela, il a été très important pour moi.

V. \& I. - Que reste-t-il de cela aujourd'hui? Qu'as-tu gardé de cette rencontre avec l'automatisme?

F. C. - J'ai gardé ce que j'appelle l'élan, une certaine forme d'automatisme que je pratique encore aujourd'hui. J'écris un peu comme un peintre. Il y a un travail de découpage et de reconstruction dans mes textes. Et chaque fois que je vais à une exposition, j'ai l'impression d'en apprendre sur la littérature. La peinture m'a appris à travailler au niveau de l'effet de surprise, à rapprocher des mots et des phrases d'une façon tout à fait libre, tout à fait gratuite et à voir ce que ça donne, comme lorsqu'on superpose deux couleurs: tout à coup une certaine profondeur, une certaine vibration apparait.

V. \& I. - Les derniers recueils créent l'impression que le réel t'échappe, devient simplement l'objet d'une expérience fugitive. Qu'est-ce finalement que le monde?

F. C. - Le monde, c'est ce qui arrive. On s'en approche à travers l'image, les mots; mais le réel résiste aux mots, est toujours beaucoup plus que les mots, il vient avant la pensée et il lui échappe toujours d'une certaine manière.

\section{V. \& I. - Pourquoi le Monde comme obstacle?}

F. C. - L'obstacle, c'est le monde de la pensée, des fantasmes. Pour arriver au monde et au réel, il faut traverser des résistances, des peurs, des illusions véhiculées par le monde de la pensée. On ne peut jamais échapper à cela une fois pour toutes parce qu'on est constamment dans un monde de représentations; et ça, ce n'est jamais la chose même.

V. \& I. - La poésie, ce serait une tentative de retrouver le contact direct avec le monde?

F. C. - C'est la volonté d'exprimer cè que j'appelle la fulgurance du réel, mais on n'y arrive jamais totalement: on ne peut que faire sentir cela.

V. \& I. - Dans l'écriture, on n'est jamais en contact direct avec le réel...?

F. C. - Jamais. C'est sans doute le drame de ma vie. J'aurais voulu être en contact direct avec le réel. J'aurais voulu nommer, parler, toucher le réel. Or c'est impossible et j'ai de la difficulté à accepter 
cela. C'est dans la voix, le cri, le lyrisme, dans ce que j'appelle l'émotion, que je me sens le plus près du réel, de l'éblouissement du réel, mais la distance demeure... et c'est tant mieux d'une certaine manière, car penser que les mots c'est le réel, ça peut être dangereux, autant sur le plan individuel que sur le plan social. Il est bon de garder une distance face au réel, à ce que l'on en perçoit et à ce que l'on en pense, bien que la fonction de la pensée soit de le célébrer.

V. \& I. - La volonté de simplification que l'on trouve dans tes derniers recueils, ça s'inscrit dans cette démarche de rapprochement de la chose même?

F. C. - C'est relié à ça et à un désir d'atteindre le lecteur d'une manière plus profonde. Je me rendais compte que les textes publiés de 1970 à 1980 rejoignaient beaucoup moins les gens et que les nouveaux lecteurs - les étudiants - étaient devenus pratiquement insensibles à ce qui s'était passé à cette époque-là; je voulais les atteindre, les toucher et leur parler des grands thèmes de la poésie qui me questionnent aussi: l'amour, la mort, Dieu... Ce langage "simple", c'était pour moi un nouveau défi; comment peut-on faire de la poésie avec des phrases qu'à la limite on retrouve dans des nouvelles, des romans, avec une prose des plus simples? J'ai découvert que c'était dans le dépouillement que les émotions et la voix passaient le mieux, contrairement à ce qu'on peut penser. C'est peutêtre dans le dépouillement que la pensée est le plus au travail. Et il est difficile d'être simple: c'est dur à atteindre, le dépouillement. Et c'est pourtant ce que recherchent le plus les peintres et les écrivains lorsqu'ils progressent dans leurs œuvres: ils visent la simplicité car c'est là que résonne le plus le corps du sujet et que la pensée tout à coup se met à rayonner. Plus je simplifie, plus l'émotion est intense, et plus la pensée me questionne, me sollicite et me déroute. Faisant cela, je voulais aussi faire bouger l'image que l'on se fait de la poésie.

V. \& I. - Y a-t-il eu effectivement élargissement du public?

F. C. - Oui. Je n'ai jamais eu autant de témoignages de lecteurs...; j'arrive à rejoindre des gens généralement peu concernés par la poésie parce que ma poésie trouve en eux une résonance beaucoup plus qu'auparavant, même s'ils ne comprennent pas nécessairement tout ce que j'écris. Ça signifie que j'ai réussi à opérer une brèche, à susciter une curiosité par rapport à l'inconnu... Pour moi, c'est extraordinaire.

V. \& I. - Peut-on encore garder, comme écrivain, une fonction critique aujourd'hui?

F. C. - Je crois que c'est non seulement possible, mais essentiel. Ma poésie reste, je crois, une poésie critique. Mes textes sont encore des textes de révolte. La beauté des visages ne pèse pas sur la terre, c'est une immense révolte contre tout ce qui empêche la poésie 
d'émerger, même si c'est relié à un désir de paix, de quiétude, de vivre profondément des relations d'amour et d'harmonie et de contemplation du monde extérieur. Pour moi, le fait d'affirmer, c'est une manifestation critique par rapport à tous les discours qui tuent le sujet, qui disent que ce qui concerne le sujet est négligeable et qu'il faut s'occuper de ce qui est utile, productif. Moi, je dis non, il faut aussi s'intéresser aux émotions, aux sentiments: être heureux, ce n'est pas seulement travailler, c'est aimer, c'est pouvoir s'exprimer, à la limite c'est pouvoir créer, peu importe le domaine d'activité dans lequel on est. Défendre le droit au bonheur, ça fait donc partie pour moi de la critique, indirectement.

V. \& I. - On a l'impression que la poésie actuelle s'est réconciliée avec sa propre histoire; on semble intégrer une certaine tradition, on admet des filiations. Que signifie cette recherche de racines?

F. C. - Effectivement, il y a une volonté de renouer avec une certaine tradition, de la relire autrement. Pour ma part j'admire beaucoup les poètes qui ont osé avant nous affronter les grandes questions qui nous hantent et qu'on a tendance à refouler. Je trouve ça courageux. Ils ont préféré faire face aux dangers plutôt que de vivre à moitié. Ils ont vécu d'une façon intense, quitte à y laisser leur peau parfois. Je trouve ça admirable. Je me sens très près par exemple de Saint-Denys Garneau dans ce que je fais. Il y a un dépouillement chez lui qui me rejoint directement, une émotion qui émerge à fleur de peau. Sauf qu'il n'a pas osé traverser sa peur du rien, du vide, en raison d'une culpabilité qui l'empêchait de vivre vraiment ses émotions et de jouir de l'existence...

V. \& I. - Vers quoi vas-tu maintenant?

F. C. - Je m'en vais vers la question de l'amour. Qu'est-ce que c'est que l'amour? C'est sans doute ce qui est à l'origine de l'histoire humaine, tant sur le plan individuel que sur le plan social, historique. C'est vraiment ce qui me sollicite actuellement.

V. \& I. - Et quelle forme cette réflexion empruntera-t-elle?

F. C. - Celle de la poésie surtout, mais il y aura aussi un journal d'accompagnement sur le lien entre l'amour et le détachement, sur le paradoxe: aimer le monde et accepter de le perdre. L'amour, donc, relié à la question du deuil.

V. \& I. - Ce sera dépouillé?

F. C. - Oui, ça va être très, très dépouillé, très métaphysique.

V. \& I. - De l'ordre de l'aphorisme?

F. C. - Oui, je me livre de plus en plus à un travail d'épuration, de condensation qui peut conduire à l'aphorisme. Mais tout cela se fera dans le mouvement même de l'écriture, de la création. 